\title{
Prevenção da iniciação do consumo do tabaco: grupo focal com jovens universitários
}

Preventing smoking experimentation: focus group with young university students

\author{
Alfredo Almeida Pina-Oliveiraa, Patrícia Spinassib, Terezinha Cristina Santana Bueno ${ }^{b}$ \\ a Enfermeiro. Doutor em Ciências pela Escola de Enfermagem da Universidade de São Paulo (EEUSP). Professor do Programa Stricto Sensu em Enfermagem da \\ Universidade de Guarulhos (UNG) e da Faculdade Campo Limpo Paulista (FACCAMP). \\ b Enfermeira. Bacharel em Enfermagem pela FACCAMP.
}

\begin{tabular}{l|l} 
RESUMO Objetivo: Compreender a percepção de elementos protetores contra a iniciação do consumo do tabaco entre jovens & Oniva
\end{tabular} universitários.

Materiais e Métodos: Pesquisa qualitativa baseada na técnica do grupo focal realizado com nove estudantes de uma faculdade particular da região metropolitana de São Paulo.

Resultados: A análise temática do discurso proporcionou duas categorias. A primeira categoria descreve os "fatores de proteção relacionados à iniciação do tabagismo" e é composta por: 'posicionar-se contra o fumar', 'evitar prejuízos estéticos e odor desagradável do tabaco' e 'reconhecer as limitações das doenças tabaco-relacionadas'. A segunda categoria engloba os "comportamentos preventivos contra o tabagismo", a saber: 'valorizar a própria saúde e ter controle sobre si', 'respeitar o conselho dos pais e ter bons exemplos' e 'participar de projetos contra drogas'.

Conclusão: Estratégias de enfrentamento do tabagismo no âmbito universitário devem incorporar a positividade dos fatores que protegem os jovens e integrar aspectos individuais, familiares e coletivos na construção de comportamentos preventivos nessa fase da vida.

Palavras-chave: comportamentos saudáveis; uso de tabaco; adulto jovem; educação superior; promoção da saúde.

Objective: To understand the perception of protective elements against the initiation of smoking among university students.

Materials and Methods: Qualitative research based on focus group including nine students from a private College in the metropolitan area of Sao Paulo, Brazil.

Results: Thematic analysis has presented two main categories. The first category is "protection factors against smoking initiation" and it is composed by 'standing up against smoking', 'avoiding esthetic harms and awful odor of tobacco' and 'recognizing limitations regarding tobacco-related diseases'. Second category embodies "preventive behaviors facing smoking" and it is made of 'valuing your own health and taking control of yourself', 'respecting parents' advices and having role models' and 'joining drug-free projects". Conclusion: Tobacco-free actions in Higher Education Institutions must incorporate positive protective factors that protect young people and integrate personal, familiar and collective aspects when building preventive behaviors at this life stage.

Keywords: health behavior; tobacco use; young adult; education, higher; health promotion.

\section{Correspondência:}

Alfredo Almeida Pina-Oliveira

Secretaria do Ambulatório Geral e Didático do Hospital das Clínicas - USP

Av. Dr. Enéas de Carvalho Aguiar, 155, 4o andar, bloco 6

05403-000 São Paulo, SP, Brasil

E-mail: alfredo.pina@hc.fm.usp.br 


\section{INTRODUÇÃO}

A juventude representa um ciclo vital oportuno para o planejamento, a implementação e a avaliação de estratégias de prevenção e de controle relacionadas ao tabagismo pela equipe de enfermagem e demais profissionais de saúde ${ }^{1,2}$

Em relação à experimentação de cigarro entre os adolescentes brasileiros, houve queda na prevalência de $24,2 \%$ em 2009 para 22,3\% em 2012. Outros produtos de tabaco fumígenos (cigarro de palha ou enrolados a mão, charuto, cachimbo, cigarrilha, cigarro indiano ou bali, narguilé) e não fumígenos (rapé e fumo de mascar) foram consumidos por $4,8 \%$ dos entrevistados 3 .

Apesar dos avanços no controle do tabagismo, o enfermeiro deve compreender seu papel estratégico na incorporação dos princípios da Convenção-Quadro de Controle do Tabaco (CQCT) e da Política Nacional de Controle do Tabagismo em defesa dos direitos dos cidadãos por ambientes livres da poluição tabagística ambiental (PTA), no reforço da ação comunitária para enfrentar os malefícios do consumo do tabaco e no acesso ao tratamento para a cessação do tabagismo nos serviços de saúde².

A etapa de transição entre o ensino médio e o ingresso nas Instituições de Ensino Superior (IES) coincide, na maioria das vezes, com o final da adolescência e início da juventude. Esse período apresenta novas experiências, aumento da liberdade individual e desenvolvimento da própria identidade adulta. Entretanto, a adoção de comportamentos negativos à saúde ocorre sobremaneira nessa etapa da vida ${ }^{4,5}$.

Desse modo, o enfermeiro pode empregar a educação em saúde aliada a ações comportamentais, culturais, institucionais e socioambientais a fim de garantir a positividade da saúde no decorrer da vida acadêmica ${ }^{6}$.

A incipiência de avaliações sobre fatores de proteção, em detrimento daquelas que enfatizam os fatores de risco relacionados ao consumo de tabaco entre jovens universitários, pode dificultar o delineamento de ações preventivas de caráter multifatorial e de estratégias baseadas na adoção de outros hábitos saudáveis em programas de Promoção da Saúde em Universidades ${ }^{7,8}$.

Pretendeu-se contribuir para reduzir essa lacuna do conhecimento por meio da seguinte questão norteadora: Quais estratégias foram empregadas por jovens universitários a fim de evitar a iniciação do consumo de tabaco?

O presente estudo foi delineado para responder a essa pergunta baseado no seguinte objetivo: compreender a percepção de elementos protetores contra a iniciação do consumo do tabaco entre jovens universitários.

\section{MATERIAIS E MÉTODOS}

Trata-se de uma pesquisa de natureza qualitativa, descritiva e exploratória, com o emprego da técnica de grupo focal definida por Kitzinger ${ }^{9: 31}$ como um "tipo de entrevista em grupo que valoriza a comunicação entre os participantes da pesquisa a fim de gerar dados" para as investigações em diferentes áreas do conhecimento.

Essa técnica de coleta de dados fundamenta-se na interação, na troca de experiências, na ampliação do repertório individual e no diálogo descontraído para o reconhecimento de conceitos, práticas, valores e atitudes empregados e compartilhados entre os participantes da pesquisa ${ }^{9,10}$.

Como cenário do estudo, optou-se por uma Instituição de Ensino Superior (IES) com fins lucrativos situada na região metropolitana do estado de São Paulo e que oferece 23 cursos de Bacharelado, 17 graduações tecnológicas e 9 cursos de Licenciatura. Entende-se que o ambiente universitário favorece a compreensão do consumo de tabaco e demais substâncias psicoativas.

Dadas às peculiaridades da logística do grupo focal ${ }^{10}$, houve o preparo de duas estudantes de Enfermagem para a abordagem em grupos, a elaboração de mensagem para o corpo docente selecionar potenciais participantes, o delineamento do guia temático com o roteiro de perguntas norteadoras, a definição da data da sessão com os estudantes e a reserva de um espaço sem interferências externas e com disponibilidade de mobiliário para organizar uma roda de conversa, boa acústica e garantia de privacidade.

A convocação para o grupo focal foi autorizada pela diretora da IES, sendo enviados e-mails institucionais ao corpo docente com a periodicidade de um mês, uma quinzena e uma semana antes de sua realização em um sábado junto às turmas de reforço acadêmico. A coleta de dados no período noturno e durante a semana não foi possível para o presente estudo devido à dinâmica das aulas.

No dia determinado, o convite foi reforçado nas salas de aula pelos três pesquisadores e os professores dos cursos de Bacharelado em Enfermagem e Farmácia, de Tecnologia em Segurança no Trabalho, de Licenciatura em Física e em História permitiram a liberação dos estudantes. Haviam 56 estudantes nesse dia, 17 manifestaram interesse e a amostra final foi constituída por 5 mulheres e 2 homens, sendo 6 graduandos do Bacharelado em Enfermagem e 1 estudante da Licenciatura em Física.

Os critérios de inclusão adotados foram: não serem fumantes atuais, serem estudantes regulares do primeiro ano do respectivo curso e serem menores de 24 anos. Realizou-se essa delimitação de faixa etária com base no conceito 
“jovem" definido pela Organização das Nações Unidas para a Educação, a Ciência e a Cultura (UNESCO) $)^{11}$ que contempla "pessoas entre as idades de 15 a 24 anos de idade" e que representa uma transição entre a adolescência e a vida adulta.

Em seguida, o grupo focal foi conduzido pelo autor principal que atua como coordenador de grupos educativos em saúde e como pesquisador qualitativo nas linhas de pesquisa sobre a formação profissional em Promoção da Saúde. O papel do coordenador foi garantir a efetiva realização das etapas de apresentação dos participantes, o treinamento das estudantes para a presente investigação, o esclarecimento de dúvidas sobre os instrumentos de coleta de dados, o desenvolvimento da sessão e a avaliação do encontro ${ }^{9}$.

As duas estudantes de Enfermagem apresentaram o Termo de Consentimento Livre e Esclarecido (TCLE) aos participantes, esclareceram as dúvidas sobre os aspectos éticos da pesquisa, aplicaram o questionário de caracterização sociodemográfica, atuaram como observadoras para a elaboração do relatório da sessão, ofereceram apoio operacional, prepararam o ambiente e os equipamentos para garantir uma boa gravação da sessão e organizaram a confraternização com os participantes ao final do encontro.

Reservou-se uma sala de aula sem interferências externas e foram utilizados dois gravadores posicionados em dois pontos opostos da formação circular das cadeiras e para explorar o objeto do presente estudo, foram apresentadas as seguintes questões norteadoras aos participantes: 1 . Como vocês percebem o tabagismo na sociedade atual? 2. Vocês já sofreram pressões para começar a fumar? 3. Quais foram suas estratégias para não iniciar o consumo do tabaco? 4. Como vocês promovem a sua saúde?

O grupo focal durou 47 minutos e foi transcrito em 3 horas e 20 minutos pelas duas estudantes de Enfermagem. O diálogo registrado possibilitou a leitura flutuante, a exploração do conteúdo e a organização dos dados, segundo as recomendações de Bardin ${ }^{12}$.

Empregou-se a análise temática da transcrição do grupo focal ${ }^{12}$. A extração de dados foi realizada pelo pesquisador principal a fim de identificar convergências e divergências no discurso dos estudantes. Os extratos grifados com diferentes cores possibilitaram a reorganização do material empírico, a seleção dos temas, a categorização e a discussão dos resultados.

A presente pesquisa foi aprovada pelo Comitê de Ética em Pesquisa da Faculdade Campo Limpo Paulista, sob o no 0041/2012 e contemplou os princípios éticos da Resolução no 466/2012 do Conselho Nacional de Saúde.

\section{RESULTADOS}

Com base na caracterização do perfil sociodemográfico, os sete participantes residem com os pais e são solteiros sem filhos. Seis estudantes são egressos de escolas públicas da região, quatro afirmam conviver com outros fumantes no domicílio e três trabalham. A média de idade dos participantes foi 20,14 anos e desvio-padrão de 0.89 .

A análise temática do material empírico transcrito possibilitou a definição de duas categorias. A primeira categoria consiste em "Fatores de proteção relacionados à iniciação do tabagismo" e foi composta pelas seguintes subcategorias: "posicionar-se contra o fumar", "evitar prejuízos estéticos e odor desagradável do tabaco" e "reconhecer as limitações das doenças tabaco-relacionadas".

A segunda categoria "Comportamentos preventivos contra o tabagismo" foi composta por três subcategorias, a saber: "valorizar a própria saúde e ter controle sobre si", "respeitar o conselho dos pais e ter bons exemplos" e "participar de projetos contra drogas".

Dada à interação entre os sujeitos de pesquisa e à unicidade grupal para responder ao objetivo da presente pesquisa $^{9,10}$, preferiu-se apresentar os extratos dos discursos sem classificações específicas para os participantes, mas cada trecho entre aspas representa falas de indivíduos distintos.

O "posicionar-se contra o fumar" reflete um discurso que enfatiza o parecer contrário ao consumo do tabaco e a atribuição de valores negativos ao fumar quando relacionado aos cuidados prestados por profissionais de saúde.

Então na verdade como o foco é idade, se for parar pra pensar, aquela pessoa que fuma, depois de uma certa idade a gente começa a associar, aos riscos, a gente começa a entender melhor [o mal] que pode causar.

Mas a palavra tabagista, você pensa na pessoa que já fuma há anos. Você não a relaciona a jovens. Se todo mundo tá fumando, a gente também vai fumar junto? Eu já penso ao contrário! Pra mim não é uma forma de diversão ou distração...

Pelo menos para nós da área da saúde é inaceitável fumar, a pessoa está ali estudando e sabendo os riscos que corre... Como o médico vai cuidar da pessoa se ele é tabagista? E o cheiro que fica depois mesmo que ele lave a mão, 'pega' o paciente com aquele cheiro?

O "evitar prejuízos estéticos e odor desagradável do tabaco" denota a importância da beleza, da higiene e do asseio pessoal no cotidiano e nos relacionamentos dos jovens universitários. 
Minha mãe sempre achou feio mulher ficar com cigarro ou uma latinha de cerveja na mão, então eu sempre tive isso pra mim sobre como isso é feio pra mulher.

Evito fumar por questões estéticas. Uma coisa que eu sempre reparei em fumantes, são os dentes, amarelados O cheiro ruim que exala pelos poros e o mau hálito... até a pele modifica, parece ficar mais grossa!

Toda hora minha mãe fuma! Mas não é por isso que eu vou fumar, porque eu tenho dois irmãos e uma irmã e um irmão e nem por isso nenhum dos dois fuma é uma coisa que me incomoda bastante, porque é só chegar perto dela que eu sinto o cheiro de cigarro. Se eu pegar na mão dela então, eu não consigo (...). Ela pode tomar banho mais o cheiro já tá nela, fica nela.

O cheiro ruim é uma coisa que marca bastante! Muitos falaram do fedor, mais tem aqueles cigarros que tem cheiro de canela, menta, né?

Ao "reconhecer as limitações das doenças tabacorelacionadas", os jovens universitários identificam os malefícios da dependência do tabaco para a própria saúde e a de outras pessoas do convívio mais próximo, assim como as dificuldades e as superações em realizar a cessação do tabagismo.

Lá em casa, a maior parte das brigas é por causa do cigarro. Meu pai era fumante e como eu tenho bronquite, eu ficava 'supermal'. Depois que ele teve o aneurisma e foi intubado, o estado dele se agravou por causa do cigarro e ai ele ficou internado um tempo. Depois quando saiu, ele fumava desde os 12 anos, ele falava que ia tentar parar. Foi difícil, mas ele parou.

Quando eu fiquei internada, minha mãe fazia assepsia, colocava touca e tudo mais para ir me ver... Então o médico disse que a partir do momento que eu tivesse alta, ela não poderia estar em contato com pessoas que fumam. Minha mãe parou [de fumar] naquele período (...). Ela voltou a fumar de novo, mas ela fuma lá fora ao ar livre, mas querendo ou não a fumaça continua.

Eu tenho medo de ficar doente ou ficar muito fraco para as outras doenças. Eu já vi várias pessoas que terminam a vida por causa do cigarro. Para mim esse é o maior sentido para nem experimentar, imagina meu pulmão ficando daquela cor [das imagens dos maços de cigarro].

Em relação aos "comportamentos preventivos contra o tabagismo", a primeira subcategoria "valorizar a própria saúde e ter controle sobre si" aponta para uma dimensão individual da proteção e das atitudes de autocuidado dos jovens universitários.
Eu acho que a parte de resistência física como subir uma escada, a pessoa que fuma fica mais cansada. Pega uma pessoa que não tem nenhum problema, nenhum tipo doença e coloca junto com um tabagista. A resistência dela é totalmente diferente".

Na minha sala de aula [turma de Enfermagem] tem muitos colegas que fumam. A gente fica tentando fazer com que a pessoa pare de fumar e quem começou recentemente [a fumar], a gente pega no pé, mas não faz muita diferença. Tem que ter opinião própria e força de vontade para não fumar. Tem que se gostar!".

A segunda subcategoria "respeitar o conselho dos pais e ter bons exemplos" configura a dimensão familiar na aquisição e no desenvolvimento de habilidades para a prevenção do tabagismo e, por extensão, envolve a rede social dos jovens universitários como suporte para prevenir a iniciação ao consumo do tabaco.

Meu pai é da geração saúde mesmo, anda de bicicleta, capacete, luva e tudo. Após se separar da minha mãe, ele sempre procura se relacionar com uma namoradinha, agora quando ele acha alguém que fuma ele descarta. Eu sou assim também!

A minha mãe já fumou quando era jovem. Um dia quando eu cheguei da balada e deixei um maço de cigarro largado no quarto, ela falou brava e firme comigo e me fez refletir muito sobre minhas atitudes (...). Ela falou uma frase que eu levo pra minha vida inteira 'a sua cabeça é o seu guia. Você vai fumar? Você pode fumar, eu não vou tirar o cigarro da sua boca e quebrar ele na sua frente, mas você tem que ter consciência do que você está fazendo. É uma coisa boa? Não. É uma coisa que vai melhorar sua saúde? Não. É uma coisa que só vai piorar a sua saúde e você tem que aprender a ver o que é bom e o ruim para você'. Cada um tem sua consciência.

A terceira subcategoria "participar de projetos contra drogas" representa uma dimensão programática que engloba a participação em serviços públicos e ou privados, as organizações não governamentais e as ações da sociedade civil organizada em prol da promoção da saúde e prevenção de doenças desde a infância e a adolescência.

Eu lembro que na minha escola [pública] tinha um projeto chamado PROERD [Programa Educacional de Resistência às Drogas e à Violência]. Entrava o cigarro, o álcool, outras drogas, entrava tudo. Aprendi como dizer não e saber mais dos riscos.

Eu já acho legal porque eu sempre estudei em escola particular e nunca teve isso, nada. Eu lembro que na pré-escola vestíamos fantasias para nossos pais pararem de fumar e não nos incentivarem a começar a fumar. 
Todo mundo pensa que na escola particular é toda certinha e na escola publica é tudo errado. Os pais e professores acham que sabemos os riscos [de fumar] e não é bem assim! Quando eu estudava na 7ạ série, a maioria da minha sala fumava.

Os resultados supracitados apresentam alinhamento com a revisão da literatura relacionada aos fatores de proteção e comportamentos preventivos de jovens universitários conforme a próxima seção.

\section{DISCUSSÃO}

O delineamento de intervenções mais efetivas para influenciar positivamente comportamentos preventivos durante a vida universitária implica na redução do consumo e no aumento do número de tentativas para a cessação do consumo do tabaco e reforça a adesão aos ambientes livres de poluição tabagística ambiental (PTA) e a aceitação de políticas e restrições nos campi pelos fumantes e não fumantes $7,8,13-15$.

Nesse sentido, as IES representam ambientes potencialmente favoráveis à saúde e que devem reforçar a participação do corpo discente, docente e técnicoadministrativo para o estabelecimento de medidas individuais e coletivas para consolidar a legislação vigente no país relacionada à proibição do consumo de tabaco em ambientes total ou parcialmente cobertos ${ }^{16}$.

Dong-Chul et al. ${ }^{13}$ demonstraram que a implementação de políticas antitabagismo em um campus universitário favoreceu a cessação do tabagismo, a percepção do uso de tabaco entre os pares acadêmicos e a adesão às normas de ambientes livres de tabaco.

As Instituições de Ensino Superior (IES) configuram espaços estratégicos para a defesa dos direitos à saúde e aumento da motivação dos jovens universitários para a adoção de comportamentos protetores à iniciação do consumo de tabaco e à exposição à PTA ${ }^{17}$.

Moradias estudantis devem ser consideradas na construção de ambientes favoráveis à saúde. Em levantamento realizado com 1595 estudantes norte-americanos, o consumo de tabaco aumenta entre os residentes de fraternidades universitárias ${ }^{4}$. No contexto brasileiro, conjuntos residenciais universitários ou "repúblicas" devem ser considerados nas estratégias individuais e coletivas que enfatizam a prevenção e o controle do tabagismo, o sexo seguro, o sono, o lazer, a atividade física e a alimentação saudável no contexto universitário.

Ações em promoção da saúde devem reconhecer os fatores de risco relacionados ao início da experimentação do tabaco, sem desconsiderar os fatores de proteção do indivíduo a fim de extrapolar para aspectos relacionados à família e aos recursos comunitários ${ }^{2}$ e integrar a abordagem de outros hábitos saudáveis na prevenção e controle do tabagismo entre jovens universitários ${ }^{7,18,19}$.

Nesse sentido, não se exclui a importância de proteger a própria saúde dos prejuízos e das doenças tabacorelacionadas e ter maior controle sobre si na evitação do consumo do tabaco conforme mencionado pelos participantes dessa pesquisa.

Moreno-Gómez et al. ${ }^{14}$ identificam a concomitância da qualidade reduzida na alimentação, na prática regular de atividade física e no consumo de álcool e tabaco entre 987 universitários espanhóis e atentam para a necessidade de delinear ações programáticas que enfatizam o bem-estar e a saúde integral dos estudantes para o treino de assertividade para o posicionamento contra o tabagismo.

Contudo, faz-se necessário avaliar a motivação dos estudantes em relação ao consumo de tabaco. O modelo transteórico para a mudança de hábitos pode favorecer essa identificação motivacional a fim de adequar as estratégias de comunicação e de educação em saúde que abordam diferentes processos de mudança para a tomada de consciência e de decisão sobre evitar o consumo eventual ou contínuo do tabaco em suas diferentes formas ${ }^{2,20}$.

A abordagem do consumo de outras formas de tabaco representa outra lacuna nas ações educativas em saúde dirigidas ao público universitário. O narguilé ou cachimbo d'água exemplifica esse desafio para os profissionais de saúde que desejam desenvolver ações programáticas com jovens universitários a fim de problematizar conceitos equivocados sobre formas mais "atrativas e ditas seguras" do consumo do tabaco ${ }^{16,21,22}$.

Associar o tabaco com aromas e sabores agradáveis representa um nó crítico para conter a iniciação do consumo do tabaco entre crianças e adolescentes, sendo que os sujeitos da presente pesquisa indicam que o odor desagradável e os aspectos estéticos contribuem para a evitação do consumo do tabaco entre eles.

Em revisão sistemática relacionada às estratégias de prevenção e controle do tabagismo entre estudantes de faculdades e universidades ${ }^{8}$, identificou-se a necessidade do levantamento dos aspectos culturais e étnicos para garantir maior efetividade na implementação de programas de promoção da saúde e da aceitação das políticas antitabagismo, de ambientes acadêmicos livres do tabaco e do aumento dos preços de produtos do tabaco.

O papel dos pais, familiares e amigos associado a outros "bons modelos" relacionados ao enfrentamento do consumo do tabaco deve ser integrado às ações de educação em saúde desenvolvidas nas IES, porém não foi identificada essa 
integração em programas de promoção da saúde dos jovens universitários na literatura analisada, implicando em medidas ambientais, normativas e de caráter comportamental individual.

Estudos qualitativos brasileiros reforçam e avançam na problematização do consumo de tabaco na juventude ao indicar a necessidade de incluir as questões de gênero ${ }^{22} \mathrm{e}$ das formas de vida e de trabalho ${ }^{23}$ para embasar o cuidado integral e equitativo de jovens universitários a fim de ajudá-los a se posicionar contra o tabaco a partir de abordagens positivas, lúdicas, participativas e cidadãs.

A inserção de jovens no Ensino Superior representa em si um fator protetor em relação à dependência do tabaco, em especial, quando associada à presença de políticas para o controle do tabaco nos campi e a participação em programas de prevenção do tabagismo baseados em diferentes níveis de escolaridade ${ }^{24}$

Os autores acima evidenciaram que jovens adultos que não frequentam IES podem apresentar maiores dificuldades de acesso a ações programáticas de prevenção e de controle do tabagismo ${ }^{24}$. A valorização dos programas preventivos de álcool, tabaco e outras drogas nos ciclos de ensino no Ensino Fundamental manifestada pelos participantes da presente pesquisa reforçam a necessidade de maior complementaridade entre intervenções com foco em adolescentes e adultos jovens que ingressam o Ensino Superior.

Infere-se que a baixa adesão dos estudantes foi desencadeada pela exigência do grupo focal ocorrer durante um sábado, pelo horário da condução da atividade investigativa (11h30 às $12 \mathrm{~h} 45)$ e pela pouca familiaridade do corpo docente e discente na participação em estudos qualitativos. Esses elementos limitaram a seleção e a composição do grupo focal.

Entende-se que a realização de um grupo focal compreende uma limitação metodológica, porém possibilitou iniciar a discussão mais específica sobre os fatores de proteção em detrimento aos fatores de risco que embasam as ações individuais e ou coletivas realizadas por enfermeiros e demais profissionais de saúde em prol da prevenção e do controle do tabagismo entre os jovens universitários.

À guisa de considerações finais, o presente estudo destaca a identificação de elementos qualitativos que extrapolam a atual discussão sobre fatores de proteção e comportamentos preventivos do tabagismo durante a juventude e, em particular, no cenário do Ensino Superior brasileiro representou a principal contribuição do presente estudo.

Compreender o que motiva a não iniciar o consumo do tabaco pode ajudar na adequação das abordagens dos profissionais de saúde que visam superar o foco nos riscos e nos prejuízos do tabagismo e que desejam reforçar atitudes individuais, familiares e coletivas contrárias à iniciação do tabagismo na juventude, sem desconsiderar os demais ciclos vitais.

A dialética entre aspectos individuais, familiares, comunitários e ambientais evidencia a historicidade e a dinamicidade sociocultural na estruturação de comportamentos preventivos adotados pelos jovens universitários na contemporaneidade, demonstrando novas tendências em sua relação com os diferentes produtos do tabaco e, por conseguinte, a necessidade de novas abordagens dos profissionais de saúde.

Espera-se que as categorias apresentadas possam suscitar novos objetos de estudo em pesquisas qualitativas e possibilitar a criação de formas de mensurar os efeitos de intervenções que enfatizam os fatores de proteção a partir de pesquisas quantitativas.

Em suma, as Instituições de Ensino Superior representam cenários oportunos e relevantes para a implementação de medidas de prevenção e de controle do tabagismo. Profissionais de saúde que desenvolvem ações no âmbito estudantil podem beneficiar-se das categorias empíricas do presente artigo para a construção de uma vida acadêmica livre do tabaco à luz da Promoção da Saúde.

\section{REFERÊNCIAS}

1. Giron MPN, Souza DP, Fulco APL. Adolescent tobacco smoking prevention: a challenge for nurses. Rev Min Enferm. 2010;14(4): 587-94.

2. Oliveira AAP, Chiesa AM. Prevenção e controle do tabagismo. In: Harada MJCS, Pedreira MLG, Viana DL, organizadoras. Promoção da saúde: fundamentos e práticas. São Caetano do Sul: Yendis; 2012. p. 399-414.

3. Brasil. Instituto Brasileiro de Geografia e Estatística. Pesquisa Nacional de Saúde do Escolar 2012 [Internet]. Rio de Janeiro: IBGE; 2012 [capturado 2016 Ago 30]. Disponível em: http://www.ibge. gov.br/home/estatistica/populacao/pense/2012/pense_2012.pdf

4. Scott-Sheldon LA, Carey KB, Carey MP. Health behavior and college students: does Greek affiliation matter? J Behav Med. 2008;31(1): 61-70. https://doi.org/10.1007/s10865-007-9136-1

5. Van Kim NA, Laska MN, Ehlinger E, Lust K, Story M. Understanding young adult physical activity, alcohol and tobacco use in community colleges and 4-year post-secondary institutions: a cross-sectional analysis of epidemiological surveillance data. BMC Public Health. 2010;26;10:208. https://doi.org/10.1186/1471-2458-10-208

6. Lenz BK. Correlates of young adult tobacco use: application of a transition framework. J Sch Nurs. 2003;19(4):232-7. https://doi.org /10.1177/10598405030190040901

7. El Ansari W, Stock C. Factors associated with smoking, quit attempts and attitudes towards total smoking bans at University: a survey of seven Universities in England, Wales and Northern Ireland. Asian Pac. J Cancer Prev. 2012;13(2):705-14. https://doi.org/10.7314/ APJCP.2012.13.2.705 
8. Murphy-Hoefer R, Griffith R, Pederson LL, Crossett L, lyer SR, Hiller $\mathrm{MD}$. A review of interventions to reduce tobacco use in Colleges and Universities. Am J Prev Med. 2005;28(2):188-200. https://doi. org/10.1016/j.amepre.2004.10.015

9. Kitzinger J. Grupos focais com usuários e profissionais da atenção à saúde. In: Pope C, Mays N, organizadores. Pesquisa qualitativa na atenção à saúde. 2ª ed. Porto Alegre: Artmed; 2005. p. 31-40.

10. Mazza VA, Melo NSFO, Chiesa AM. Focal group as a data gathering technique in qualitative research: experience report. Cogitare Enferm. 2009;14(1):183-8.

11. United Nations Educational, Scientific and Cultural Organization. What do we mean by "youth"? [Internet]. London: University of London, Queen Mary, Department of Chemistry; 2014 [atualizado 2006 Jul 24; citado 2016 Ago 30]. Disponível em: http://www. unesco.org/new/en/social-and-human-sciences/themes/youth/ youth-definition/

12. Bardin L. Análise de conteúdo. Lisboa: Edições 70; 2010.

13. Seo DC, Macy JT, Torabi MR, Middlestadt SE. The effect of a smoke-free campus policy on college students' smoking behaviors and attitudes. Prev Med. 2011;53(4-5):347-52. https://doi.org/ 10.1016/j.ypmed.2011.07.015

14. Moreno-Gómez C, Romaguera-Bosch D, Tauler-Riera P, BennasarVeny M, Pericas-Beltran J, Martinez-Andreu S, Aguilo-Pons A. Clustering of lifestyle factors in Spanish university students: the relationship between smoking, alcohol consumption, physical activity and diet quality. Public Health Nutr. 2012;15(11):2131-9. https://doi.org/10.1017/S1368980012000080

15. Salazar-Torres IC, Varela-Arévalo MT, Lema-Soto LF, TamayoCardona JA, Duarte-Alarcón C, Equipo de investigación CEVJU Colombia. Assessing young university students' behaviour regarding health. Rev Salud Pública. 2010;12(4):599-611.

16. Brasil. Ministério da Saúde. Agência Nacional de Vigilância Sanitária. Instrução Normativa № 6, de 26 de Agosto de 2013. Regra atualizada sobre aditivo de tabaco. DOU. 201327 Ago.

17. Yang T, Abdullah AS, Rockett IR, Li M, Zhou Y, Ma J, Ji H, Zheng J, Zhang Y, Wang L. Assessment of tobacco control advocacy behavioural capacity among students at schools of public health in China. Tob Control. 2011;20(1):20-5. https://doi.org/10.1136/ tc. 2010.036590

18. Mantilla-Toloza SC, Gómez-Conesa A, Hidalgo-Montesinos MD. Physical activity and tobacco and alcohol use in a group of university students. Rev Salud Pública. 2011;13(5):748-58. https:// doi.org/10.1590/S0124-00642011000500003

19. Quintiliani L, Allen J, Marino M, Kelly-Weeder S, Li Y. Multiple health behavior clusters among female college students. Patient Educ Couns. 2010;79(1):134-7. https://doi.org/10.1016/j. pec.2009.08.007

20. Choi Y, Choi SM, Rifon N. "I smoke but I am not a smoker": phantom smokers and the discrepancy between self-identity and behavior. J Am Coll Health. 2010;59(2):117-25. https://doi.org/10.1080/0744 8481.2010.483704

21. Martins SR, Paceli RB, Bussacos MA, Fernandes FLA, Prado GF, Lombardi EMS. Experimentation with and knowledge regarding water-pipe tobacco smoking among medical students at a major university in Brazil. J Bras Pneumol. 2014;40(2):102-10. https://doi. org/10.1590/S1806-37132014000200002

22. Nuzzo E, Shensa A, Kim KH, Fine MJ, Barnett TE, Cook R, Primack BA. Associations between hookah tobacco smoking knowledge and hookah smoking behavior among US college students. Health Educ Res. 2013;28(1):92-100. https://doi.org/10.1093/her/cys095

23. Colares V, Franca C, Gonzalez E. Health-related behavior in a sample of Brazilian college students: gender differences Cad Saúde Pública. 2009;25(3):521-8. https://doi.org/10.1590/S0102$311 \times 2009000300007$

24. Panaino EF, Soares CB, Campos CMS. Contextos de início do consumo de tabaco em diferentes grupos sociais. Rev Latino-Am Enfermagem. 2014;22(3):379-85. https://doi.org/10.1590/01041169.3205 .2427

25. Lenk K, Rode P, Fabian L, Bernat D, Klein E, Forster J. Cigarette use among young adults: Comparisons between two year college students, four-year college students, and those not in college. J Am Coll Health. 2012;60(4):303-8. https://doi.org/10.1080/07448481. 2011.607481 